\title{
Disentangling syntactic, semantic and pragmatic impairments in ASD: Elicited production of passives
}

\author{
Ben AMBRIDGE ${ }^{1 *}$ (D), Amy BIDGOOD² (D), and Kate THOMAS ${ }^{3}$ \\ ${ }^{1}$ University of Liverpool, UK ESRC International Centre for Language and Communicative Development \\ (LuCiD), ${ }^{2}$ University of Salford, UK, and ${ }^{3}$ University of Liverpool, UK \\ ${ }^{*}$ Corresponding author: Ben Ambridge The University of Liverpool - Psychology Eleanor Rathbone \\ Building, Bedford St South, Liverpool L69 7ZA, UK Ben.Ambridge@Liverpool.ac.uk
}

(Received 21 February 2019; revised 17 October 2019; accepted 5 March 2020;

first published online 14 May 2020)

\begin{abstract}
Children with ASD and an IQ-matched control group of typically developing (TD) children completed an elicited-production task which encouraged the production of reversible passive sentences (e.g., "Bob was hit by Wendy"). Although the two groups showed similar levels of correct production, the ASD group produced a significantly greater number of "reversal" errors (e.g., "Wendy was hit by Bob", when, in fact Wendy hit Bob) than the TD group (who, when they did not produce correct passives, instead generally produced semantically appropriate actives; e.g., "Wendy hit Bob"). These findings suggest that the more formal elements of syntax are spared relative to more semantic/pragmatic/narrative aspects (e.g., manipulating thematic roles) in at least high-functioning children with ASD.
\end{abstract}

\section{Introduction}

Autism Spectrum Disorders (ASD) are currently thought to affect somewhere between $1 \%$ and $2 \%$ of primary/elementary school aged children (4 or 5-11) in both the UK and the USA (Baird, Simonoff, Pickles, Chandler, Loucas, Meldrum et al., 2006; Baron-Cohen, Scott, Allison, William, Bolton, Matthews et al., 2009; Kogan, Blumberg, Schieve, Boyle, Perrin, Ghandour, Singh, Strickland, Trevathan \& Van Dyck, 2009; Zaroff \& Uhm, 2011). Delayed language acquisition is almost universal amongst children with ASD; though, interestingly, in DSM-V, language impairment became an "accompanying" disorder, rather than a core diagnostic criterion. This change perhaps reflects that fact that the degree of language impairment seen in ASD varies enormously, from children who do not speak at all to those whose language development appears to be unimpaired (e.g., Kjelgaard \& Tager-Flusberg, 2001; Roberts, Rice \& Tager-Flusberg, 2004; Tager-Flusberg, Paul \& Lord, 2005; Siller \& Sigman, 2008). 
A widely-held view in the literature is that, despite their broader linguistic and communicative difficulties, "pure syntax" is relatively spared in children with ASD (or, at least, a high-functioning subgroup); i.e., spared relative to the general cognitive deficits that accompany the disorder. For example, Swensen, Kelley, Fein and Naigles (2007), Naigles, Kelty, Jaffery and Fein (2011) and Tek, Mesite, Fein and Naigles (2014) found that children with ASD showed similar patterns of acquisition to IQ-matched typically developing (TD) children with respect to morphosyntax, basic SUBJECT VERB OBJECT word order and wh-questions (see also Tager-Flusberg, 1981, 1985; Tager-Flusberg, Calkins, Nolin, Baumberger, Anderson \& Chadwick-Dias, 1990; Roberts, Mirrett \& Burchinal, 2001; but see Rapin \& Dunn, 2003; Eigsti, Bennetto \& Dadlani, 2007; Eigsti \& Bennetto, 2009; Eigsti, de Marchena, Schuh \& Kelley, 2011).

On this view, which can be summarized as "Form is easy, meaning is hard" (Naigles, 2002; Naigles \& Tek, 2017), syntax itself is spared, and the communicative difficulties that are experienced by children with ASD are caused by impairments in other areas of language such as vocabulary, semantics, socio-pragmatics and narrative (e.g., Tager-Flusberg, Lord \& Paul, 1997; Jordan, 1993). To be clear, there is evidence that even typically developing children find certain semantic/pragmatic aspects of language more difficult than purely syntactic/structural ones (Naigles, 2002); the claim is that this is even more true for children with ASD. A difficulty in testing this claim lies with the fact that syntax does not operate in a vacuum. Rather, syntactic constructions are used to convey semantic messages for (mainly) socio-pragmatic purposes. Thus any investigation of spared versus impaired aspects of language in ASD faces difficulty in picking apart these interrelated competencies.

One domain that has proved particularly useful in this regard is the acquisition of the (English) passive. This construction is useful for distinguishing syntactic and semantic/pragmatic impairments because it is a syntactic construction that, compared with canonical active sentences, reverses both the order of thematic roles (e.g., [PATIENT] [ACTION][AGENT] as opposed to [AGENT] [ACTION] [PATIENT]) and the link between these roles and conventional discourse-pragmatic functions: in a passive, the [PATIENT] rather than the [AGENT] serves as the TOPIC about which a COMMENT is made (e.g., You know Bob? Well, he was hit by Wendy [vs he hit Wendy]; e.g., Pullum, 2014).

Thus if the language of children with ASD is spared with regard to "pure" syntax, but impaired with the more semantic/pragmatic aspects of language, we might expect these children to show excellent command of the syntax of the passive, but not of the mapping between syntactic and semantic thematic roles. That is, they might be expected to perform similarly to IQ-matched typically developing (TD) children with regard to their ability to produce passive syntax (e.g., [SUBJECT] was [VERB]ed by [OBJECT]), but to incorrectly link [SUBJECT] to [AGENT] and [OBJECT] to [PATIENT] rather than vice versa, particularly for reversible passives. For example, a child with ASD might produce a syntactically well-formed passive such as Bob was hit by Wendy, with the meaning that 'Bob hit Wendy' and/or make parallel mistakes in comprehension.

Previous studies suggest some support for this prediction, though findings are mixed. Tager-Flusberg (1981) compared the performance of an ASD group (mean age $8 ; 1$ ) and an IQ-matched TD group (mean age 3;10) on an act-out task with passive (and active) sentences. Although the ASD group showed lower overall levels of correct performance, they were no more likely than the TD group to use a word-order strategy that results in incorrect "reversals" (e.g., enacting The girl is pushed by the boy as 'The girl pushes the boy'). However, one important difference 
was that only the TD children used a "probable event" strategy: TD children were more likely to enact reversals for improbable passives like The girl is carried by the baby than for reversible passives like The girl is pushed by the boy. The ASD group were equally likely to enact reversals for both sentence types.

On the other hand, Paul, Fisher and Cohen (1988) - using an almost identical set of stimuli - found that $83 \%$ of ASD children (5/6) showed evidence of using a word-order strategy for passives (resulting in incorrect reversals), as compared to only $57 \%, 50 \%$ and $25 \%$ of language impaired children (4/7), TD 2-year olds (4/8) and TD 3 -year-olds (2/8) respectively. Although only the comparison between the ASD group and the TD 3-year-olds reached statistical significance, this is almost certainly a consequence of the very small sample size. Thus, on the basis of this study at least, children with ASD do seem to be making the types of reversal errors with passives that one would expect if they show impairments in semantics, in particular the relationship between semantic and syntactic roles

Turning now to production studies, Allen, Haywood, Rajendran and Branigan (2011) found that ASD children (mean age 10;6) did not differ from either age-matched or IQ-matched (mean age 7;6) TD controls in a production priming task (see also Slocombe, Alvarez, Branigan, Jellema, Burnett, Fischer, Garrod \& Levita, 2013, for a similar finding for adults with Asperger's syndrome, and Hopkins, Yuill \& Keller, 2016, for evidence that children with ASD also show syntactic alignment in natural conversation). That is, children were more likely to produce passive picture-descriptions (e.g., The queen is being kissed by the sheep) after hearing passive (e.g., The robber is being chased by the dog) than active prime sentences (e.g., The dog is chasing the robber) - and vice versa - and the magnitude of this "syntactic alignment" effect did not differ across the three groups. In this study, however, reversals (e.g., The queen is being kissed by the sheep for a picture in which the queen is kissing the sheep) were excluded as "missing data" and not reported or analysed separately (presumably because the authors were interested mainly in comparing the extent of syntactic alignment across the three groups). Thus, while children with ASD showed similar levels of correct passive production to an IQ-matched TD group, we do not know whether or not they produced more reversals.

The aim of the present study is to investigate this possibility. On the assumption that the syntax - but not the semantics - of the passive is spared in ASD, we predict that children with ASD will show similar levels of correct passive production to IQ-matched TD children, but higher levels of reversal errors. Because we are interested in comparing the groups on reversal errors for passives - rather than the magnitude of syntactic alignment - we did not attempt to elicit actives. Rather, in order to maximize the overall number of passives produced-and hence the likelihood of observing between-group differences - a passive sentence constituted both the prime and intended target on every trial. That is, the present study does not attempt to measure the magnitude of any syntactic priming effect, and hence is not a "syntactic priming study" in the usual sense. Rather, it is an elicted production study that uses the well-established phenomenon of syntactic priming as a way to elicit passives (a very low frequency structure that children are otherwise unlikely to produce).

One further modification is that the present study also manipulated semantic verb type: agent-patient (e.g., hit), theme-experiencer (e.g., frighten), experiencer-theme (e.g., see). Although the reasons why remain controversial (and - fortunately - are not 
relevant for our purposes), the findings of many previous studies suggest that typically-developing children show particular difficulties with experiencer-theme passives (Horgan, 1978; Fox \& Grodzinksky, 1998; Gordon \& Chafetz, 1990; Hirsch \& Wexler, 2006; Maratsos, Fox, Becker \& Chalkley, 1985; Sudhalter \& Braine, 1985; Meints, 1999; Messenger, Branigan, McLean \& Sorace, 2012). Thus this manipulation allows for investigation of grammar sparing in ASD with respect to another aspect of passive production that straddles the syntax-semantics interface: if TD children show different performance for three semantically-defined verb classes with identical passive syntax, do ASD children do likewise?

\section{Method}

\section{Participants}

The Autism Spectrum Disorders (ASD) group consisted of 15 children aged 6-9 $(\mathrm{M}=$ $7 ; 11, \mathrm{SD}=0 ; 1)$. Although a larger sample size would have been desirable, it is important to note that this is a very difficult to reach population, and - as such - this sample size is in fact towards the upper end of those obtained in comparable studies $(N=6$ in Paul et al., 1988; $N=12$ in Allen et al., 2011; $N=18$ in Tager-Flusberg et al., 1981). All children in this group were recruited from specialist schools in the North West of England and had a primary diagnosis of ASD prior to enrolment. It was not possible for us to independently confirm diagnosis. However, all of these children had undergone an intensive diagnosis procedure (far more detailed than any we could have conducted), summarized in Appendix A. It is important to note that even studies specifically concerned with estimating prevalence of ASD routinely assume that false positives amongst children attending specialist schools are almost non-existent, given that, in the UK, admission to such a school requires this type of detailed team-based assessment. For example, in Baron-Cohen et al. (2009: 503) - the gold-standard of UK-prevalence studies with over 700 citations - "all cases from special schools were assumed confirmed since entry to a special school would require extensive assessment of individual special needs". The IQ-matched Typically Developing (TD) control group consisted of 15 children aged $4-5(\mathrm{M}=5 ; 1, \mathrm{SD}=$ $0 ; 8)$. All were recruited from mainstream nurseries or primary schools across England.

\section{Standardized Test}

In order to ensure IQ matching of the ASD and TD groups, each child completed the short version of the Wechsler Preschool and Primary Scale of Intelligence, Fourth Edition (WPPSI-IV) (Wechsler, 2012), which consists of seven subtests (Receptive Vocabulary, Block Design, Picture Memory, Information, Object Assembly, Zoo Locations and Picture Naming) and three subscales (Verbal Comprehension, Visual Spatial, Working Memory; see Table 1 for details). The short version of the test was used, as the full version is not recommended for children younger than 5;0. Visual inspection of these means confirms that the groups were well matched on all subtests except Object Assembly and Picture Memory, for which the ASD group showed a small advantage. Technically speaking, it is statistically inappropriate to compare the groups using inferential statistics (Sassenhangen \& Alday, 2016). Rather, we include a composite IQ measure as a control predictor in the statistical analyses (by converting all test scores into SD units and taking the mean). Nevertheless, because the practice is almost ubiquitous in 
Table 1. Performance of children in the ASD and TD groups on the WPPSI-IV

\begin{tabular}{|c|c|c|c|c|c|c|c|}
\hline & Comparison & $\begin{array}{l}t 95 \% \\
\mathrm{Cl} \text { Min }\end{array}$ & $\begin{array}{l}t 95 \% \\
\mathrm{Cl} \text { Max }\end{array}$ & Mean ASD & $S D A S D$ & Mean TD & $S D$ TD \\
\hline Vocabulary & $\begin{array}{l}t=-0.39 \\
d f=26.26 \\
p=0.6987\end{array}$ & -2.92 & 1.98 & 22.47 & 3.66 & 22.93 & 2.81 \\
\hline Blocks & $\begin{array}{l}t=-0.39 \\
d f=17.34 \\
p=0.7037\end{array}$ & -5.16 & 3.56 & 19.47 & 7.57 & 20.27 & 2.63 \\
\hline Pictures & $\begin{array}{l}t=2.04 \\
d f=24.08 \\
p=0.0529\end{array}$ & -0.06 & 8.59 & 19.33 & 6.80 & 15.07 & 4.43 \\
\hline Information & $\begin{array}{l}t=-1.74 \\
d f=22.06 \\
p=0.09624\end{array}$ & -4.83 & 0.43 & 18.53 & 4.27 & 20.73 & 2.40 \\
\hline Assembly & $\begin{array}{l}t=2.10 \\
d f=27.66 \\
p=0.04464\end{array}$ & 0.14 & 11.06 & 27.67 & 6.87 & 22.07 & 7.69 \\
\hline Zoo & $\begin{array}{l}t=-1.24 \\
d f=17.95 \\
p=0.2304\end{array}$ & -2.51 & 0.65 & 10.13 & 2.72 & 11.07 & 1.03 \\
\hline Naming & $\begin{array}{l}t=0.20, \\
d f=27.6, \\
p=0.8429\end{array}$ & -1.85 & 2.25 & 17.40 & 2.90 & 17.20 & 2.57 \\
\hline
\end{tabular}

the literature, we decided to compare the groups using a series of independent $t$ tests (Welch's test assuming unequal variances); see Table 1 . These confirmed that the two groups did not differ on any subtest except Object Assembly, in favour of the ASD group.

\section{Design and materials}

A $3 \times 2$ mixed design was used with the within-subjects factor of verb type (agent-patient, theme-experiencer, experiencer-theme) and the between-subjects factor of group (ASD, TD). 36 target verbs were used; 12 of each type:

- Agent-patient verbs: Bite, Carry, Chase, Dress, Hit, Hug, Kick, Pat, Pull, Push, Squash, Wash

- Experiencer-theme verbs: Forget, Hate, Hear, Ignore, Know, Like, Love, Remember, See, Smell, Understand, Watch

- Theme-experiencer verbs: Amaze, Annoy, Bother, Frighten, Impress, Please, Scare, Shock, Surprise, Tease, Upset, Worry

Each child completed 18 trials (plus three warm-up trials), 6 with each of the three target verb types (i.e., half of the total design), randomly distributed across children. A further 24 verbs, all agent-patient, were selected for use in prime sentences:

- Prime verbs: Avoid, Bite, Call, Carry, Chase, Cut, Dress, Drop, Eat, Follow, Help, Hit, Hold, Hug, Kick, Kiss, Lead, Pat, Pull, Push, Shake, Squash, Teach, Wash 
Note that although these prime verbs overlap with the agent-patient target verbs, no verb was used as both a prime and a target verb for any individual child (thus the need for a total set 24 prime verbs, even though each child completed only 18 trials).

For each verb, we created either a prime sentence or an intended target sentence, each using a pair of well-known children's cartoon characters: Bob and Wendy or Homer and Marge. All sentences were of the form [X] was [VERB]ed by. Within each pair, both characters appeared equally often as SUBJECT and OBJECT. The pairing between prime and target sentences was different for each participant and was pseudo-random, such that a different pair of characters was used for the prime and target sentence on each trial (i.e., the confederate's sentence used Bob and Wendy and the child's Homer and Marge, or vice versa). This ensured (a) that any by-verb differences in performance cannot be a function of the prime verb with which a particular target verb was paired (which differed across participants) and (b) that participants could not use character-specific strings or strategies from the prime sentence when producing the subsequent target sentence (e.g., Bob was VERBed by Wendy). For each sentence, a corresponding animation was created using Anime Studio Pro. The advantage of using animations, as opposed to still pictures, is that it is easier to accurately depict "who is doing what to whom" in each sentence.

\section{Procedure}

All testing was conducted individually in a quiet room or quiet area of the classroom. The WPPSI-IV test was completed over two separate days, with each session lasting between 20 and 30 minutes per child. On day three, children completed the production priming test. The experiment was run as a bingo game with the child playing against an adult confederate (e.g., Rowland, Chang, Ambridge, Pine \& Lieven, 2012). It was explained to the child that she and her opponent would take it in turns to describe an animation seen on the computer screen, using the clue word given to them by the experimenter (the target verb). The confederate took the first turn in each game, in order to initiate the sequence of passive-prime/target pairs. After each description, the experimenter, who could not see the animation, "looked to see" if she had the corresponding card (which showed a still from the animation) and, if so, gave it to the relevant player. The game was structured so that the child would not receive a card on every trial, so as to maintain suspense, but would win each game on the final trial. Children's responses were noted by hand, and subsequently verified against audio recordings.

\section{Scoring}

Children's responses were coded as follows (for the example animation 'Wendy hit Bob', total $\mathrm{N}=540$ trials: 30 children $\mathrm{x} 18$ target sentences).

- Correct passive: $\mathbf{N}=\mathbf{8 1}$. (e.g., Bob was hit by Wendy). The use of pronouns or generic NPs (e.g., He was hit by the woman) was allowed, provided that the sentence was unambiguous, but was very rare, presumably because the children knew the characters well, and heard their names used many times throughout the study.

- Reversed passive: $\mathbf{N}=\mathbf{4 2}$ (e.g., Wendy was hit by Bob, She was hit by him etc.).

- Correct active: $\mathbf{N}=\mathbf{2 3 8}$ (e.g., Wendy hit Bob). No child produced a reversed active (e.g., Bob hit Wendy). 
- Incorrect (other use of target verb): $\mathbf{N}=46$. (e.g., hit Bob) Responses in which children used the target verb but not in a correct passive, reversed passive or active were scored as incorrect, and retained in the statistical analysis.

- Excluded: $\mathbf{N}=133$ (e.g., Wendy punched Bob). Trials for which children used a non-target verb, or gave an irrelevant or no response $(N=133)$ were excluded from all statistical analyses.

Overall, then, children produced a syntactically well-formed sentence with the target verb (which they were given) on $67 \%$ of trials $(81+42+238 / 540)$. Although passives were produced only around half as often as actives, this still represents a large priming effect, given that every animation can be appropriately and straightforwardly described using an active sentence. Thus we were largely successful in our aim of maximizing the number of passives produced, in order to better observe any underlying difference in passive performance between the ASD and TD groups.

\section{Results}

All data and code can be found on the website of the Open Science Framework https:// osf.io/7tkwn/. Because the number of participants is low relative to the complexity of the model (which includes an interaction of Group by verb-type, as well as a main effect of IQ), a frequentist model would not converge with anything close to maximal random effects structure (in the sense of Barr, Levy, Scheepers \& Tily, 2014). The ability to include maximal random effects structure is crucial since non-maximal models are anti-conservative (indeed, one recent paper was retracted when an apparent effect disappeared under a maximal model; Fisher, Hahn, DeBruine \& Jones, 2015). Therefore, the data were analysed using Bayesian mixed effects models (brms R package, Bürkner, 2017) which are considerably more robust with regard to convergence failure. A conservative prior of $M=0, S D=2.77$ was chosen to correspond to what we estimated to be the maximum feasible performance change caused by differences between Groups (ASD/TD) and between verb-types (Agent-Patient/Experiencer-Theme/Theme-Experiencer); a shift from $20 \%$ to $80 \%$ correct performance:

$$
\log (0.8 / 0.2)-\log (0.2 / 0.8)=2.77
$$

This prior proved to be sufficiently wide (and hence conservative), since none of the effects observed were as large. The use of Bayesian models (with adapt-delta set to 0.95) allowed us to use maximal random effects structure (Barr et al., 2014), and to include the composite IQ score as a control predictor, as per the following brms syntax:

$$
\begin{aligned}
& \text { brm(formula }=\text { CorrectPassive } \sim(1+\text { Group }+ \text { IQ } \mid \text { Target }) \\
& +(1+\text { VerbType|Participant }) \\
& + \text { Group } * \text { VerbType }+ \text { IQ, data }=\text { Data, family } \\
& =\text { bernoulli }(), \text { set_prior(" } \text { normal }(0,2.77) ", \text { class }=\text { " } b "), \\
& \text { cores }=4, \text { warmup }=2000, \text { iter }=5000, \text { chains }=4, \\
& \text { control }=\text { list }(\text { adapt_delta }=0.95))
\end{aligned}
$$




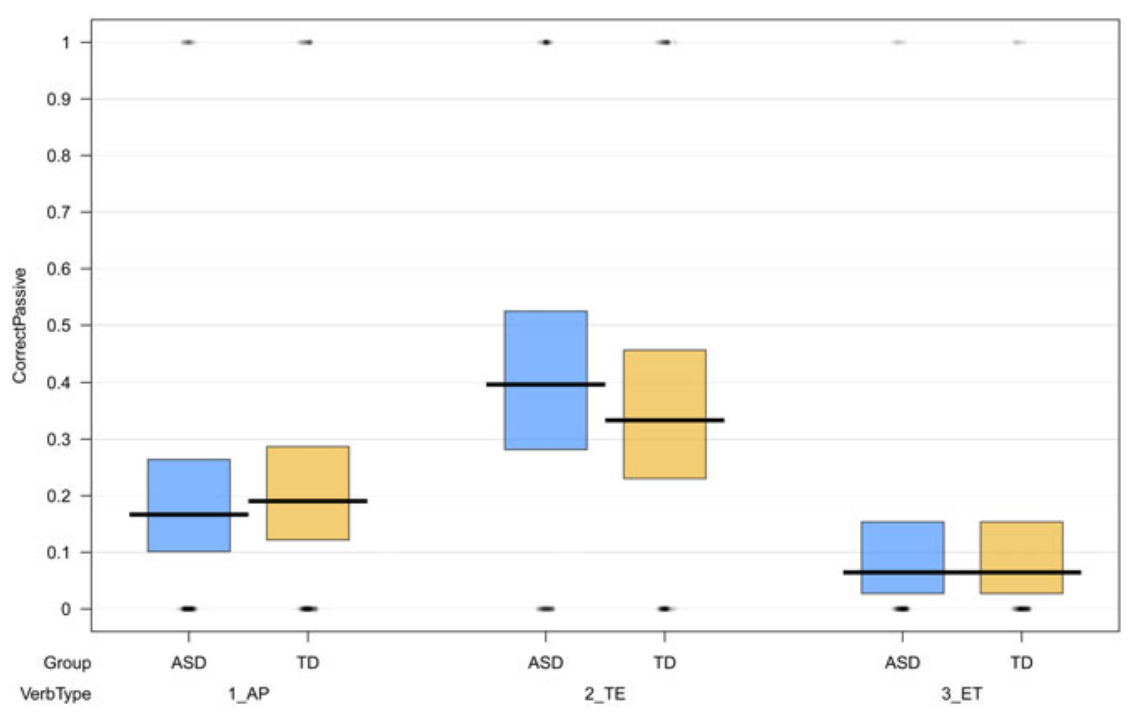

Figure 1. Correct Passives

For the predictor of Verb Type, Agent-Patient was chosen as the reference category, because these actional (as opposed to "psychological") passives are usually held to be the most "basic" type (Horgan, 1978; Fox \& Grodzinksky, 1998; Gordon \& Chafetz, 1990; Hirsch \& Wexler, 2006; Maratsos et al., 1985; Sudhalter \& Braine, 1985; Meints, 1999; Messenger et al., 2012). For the predictor of Group (ASD/TD), the former was arbitrarily chosen as the reference category on the basis of alphabetical order.

Finally, we note a further advantage of adopting a Bayesian approach: Bayesian models yield " $p$ " values ( $p M C M C$ values) and credible intervals (cf. frequentist confidence intervals) that, unlike their frequentist counterparts, can be interpreted intuitively: the $P M C M C$ value represents the probability that the true size of the effect is (for positive effects) zero or lower (for negative effects, zero or higher). The 95\% credible interval represents an interval which contains, with 95\% probability, the true value of the effect in question.

The dependent variable (1/0) was, depending on the analysis, (a) correct passives, (b) reversed passives, (c) correct actives, or (d) incorrect ("other") verb uses (with "excluded" responses treated as missing data).

\section{Correct passives}

Figure 1 illustrates the correct production of Agent-Patient, Theme-Experiencer and Experiencer-Theme passives by children in the ASD and TD group. Solid bars show mean group performance; the shaded boxes show 95\% Bayesian Highest Density Intervals (HDIs). It is clear from inspection of this plot that the ASD and TD groups show an almost identical pattern performance. Indeed, the Bayesian model (Figure 5) revealed no effect of Group, but a main effect of verb-type (as illustrated by the fact that the 95 credible intervals for VerbType2_TE and VerbType3_ET do not overlap zero). As compared to the reference category of agent-patient (hit-type) verbs $(M=$ 


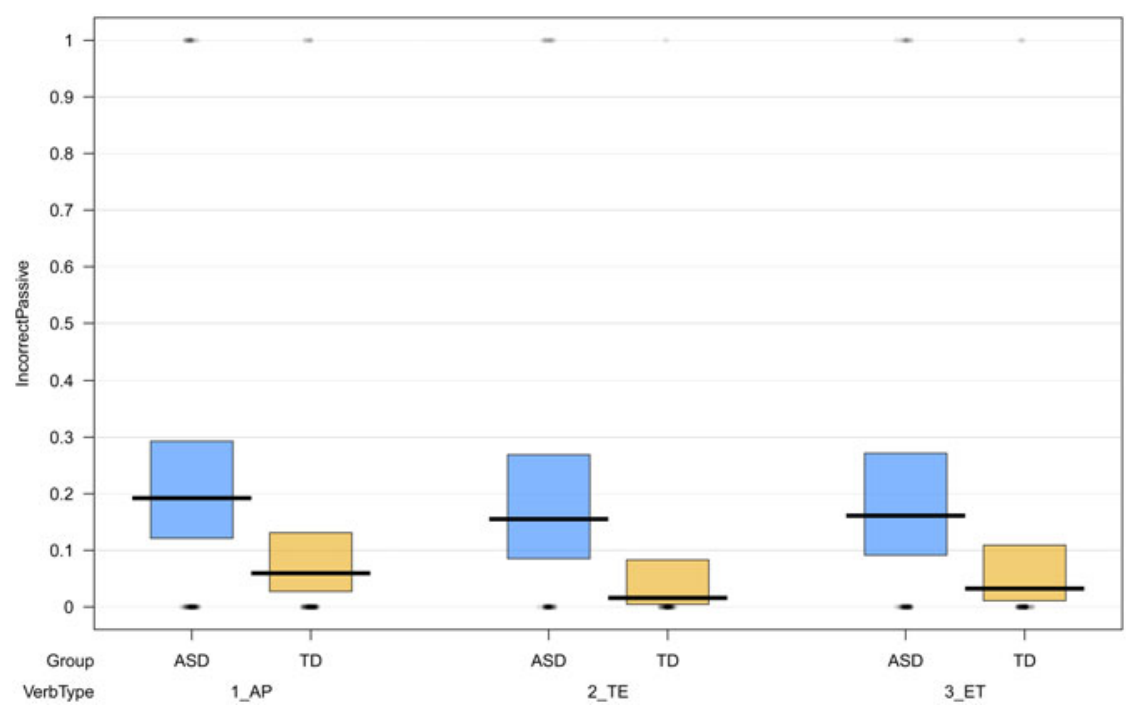

Figure 2. Reversed Passives

0.17 , correct passives; $S D=0.28)$, theme-experiencer (frighten-type) verbs $(M=0.36$, $S D=0.48)$ and experiencer-theme (see-type) verbs $(M=0.06, S D=0.25)$ showed higher $(p M C M C=0.01)$ and lower $(p M C M C=0.02)$ levels of correct passive production respectively. No interaction was observed, reflecting the fact that - as is clear from Figure 1 - the pattern described above (theme-experiencer $>$ agent-patient $>$ experiencer-theme) applies equally to the ASD and TD groups. Thus this analysis confirms the finding of previous studies that ASD and IQ-matched TD children show comparable performance with passives, and extends this finding by showing that the similarity extends to an almost-identical pattern of by-verb differences.

\section{Reversed passives}

Figure 2 illustrates the production of reversed passives by children in the ASD and TD group. The Bayesian model (Figure 6) revealed no effect of verb type, and no interaction, but - crucially - a main effect of Group: as expected, children in the ASD group produced more reversed passives $(M=0.17, S D=0.37)$ than children in the IQ-matched TD group $(M=0.04, S D=0.19 ; p M C M C=0.03)$, who produced these forms extremely rarely. Indeed, $9 / 15$ children in the ASD group produced at least one reversed passive, as compared to just 5/15 in the TD group, with four of those five producing only one. The lack of a main effect or interaction for Verb Type suggests that for neither the ASD or TD group do these reversal errors pattern according to verb type.

\section{Correct actives}

Figure 3 illustrates the production of correct (i.e., grammatically well-formed and semantically appropriate) actives by children in the ASD and TD group. The 


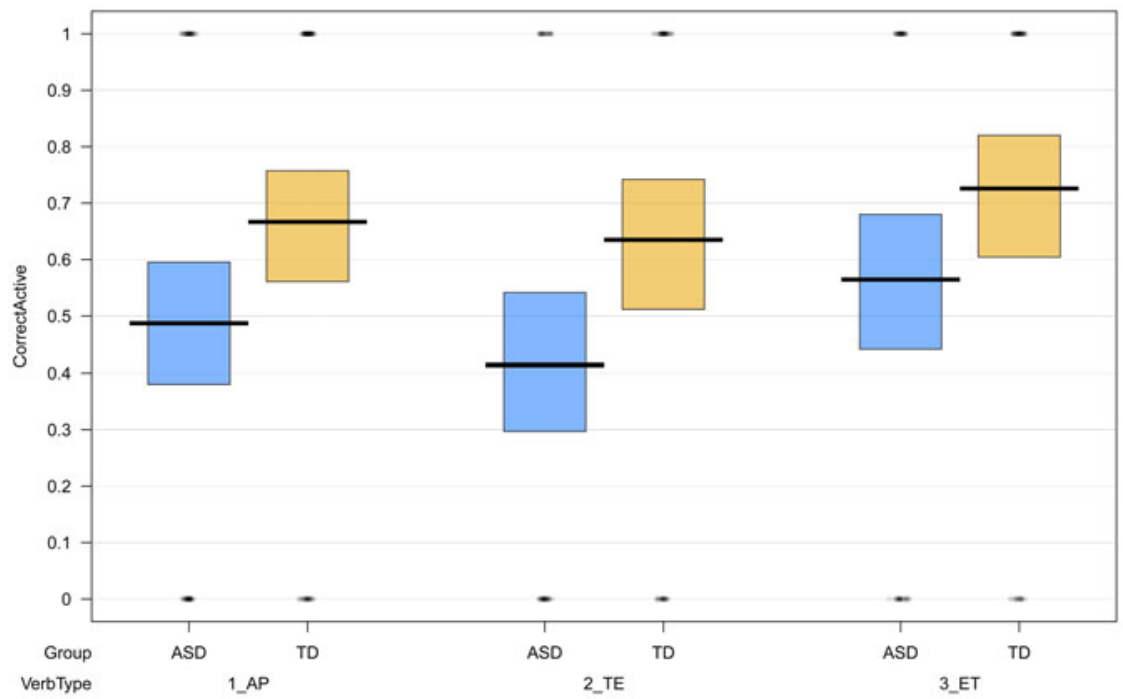

Figure 3. Correct Actives

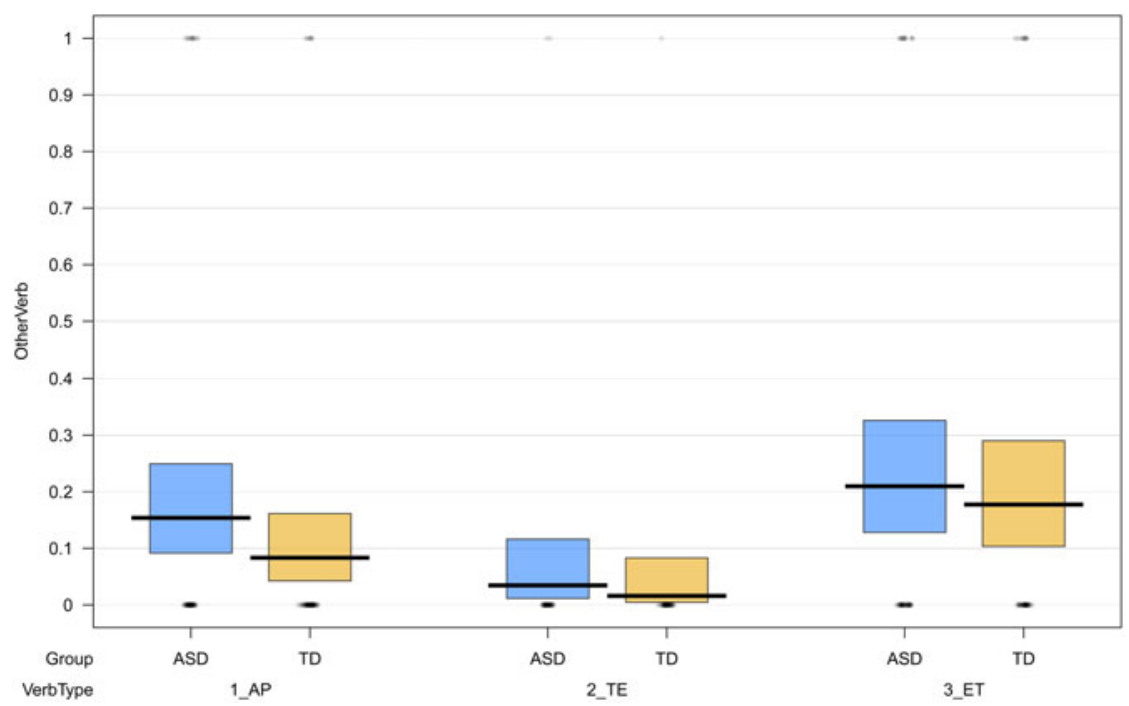

Figure 4. Other Verb Uses

Bayesian model (Figure 7) revealed suggestive evidence for a weak effect of Group, with the $95 \%$ Credible Interval overlapping zero by a very narrow margin $(p M C M C=0.058)$. Although, unlike frequentist $p$ values, Bayesian $p M C M C$ values can be interpreted probabilistically as weak evidence for an effect, nothing of importance hinges on this finding. It appears to reflect a trade-off effect such that, since they rarely produce erroneous passives, children in the TD group are more likely to produce semantically appropriate actives $(M=0.67, S D=0.47)$ than are children in the ASD group 
Figure 5. Bayesian model for Correct Passives

Figure 6. Bayesian model for Reversed Passives
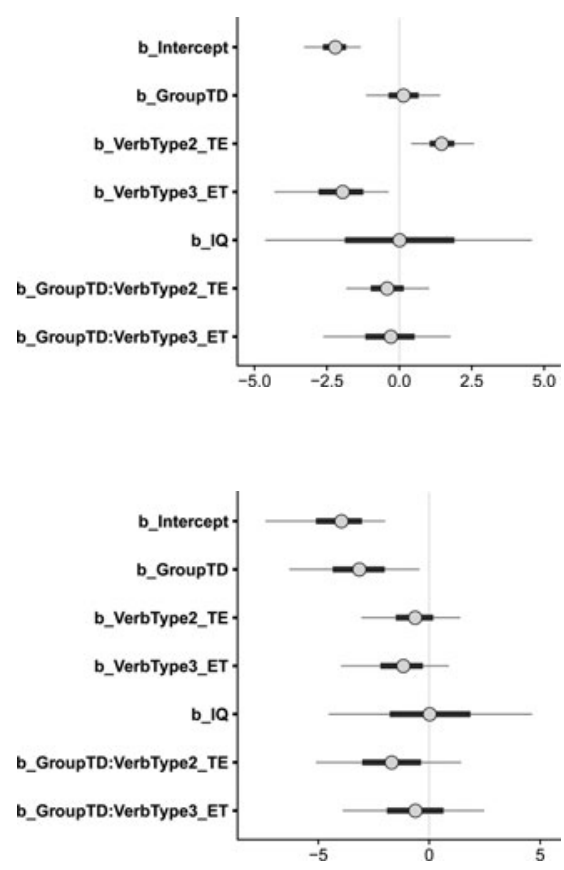

$(M=0.49, S D=0.50)$. In other words, the ASD and TD group produce correct passives at a similar rate; but where children in the former group produce reversed passives, children in the latter group produce well-formed actives. The lack of a main effect or interaction for Verb Type suggests that, for neither the ASD or TD group do these well-formed actives pattern according to verb type.

\section{Incorrect (other verb) uses}

Figure 3 illustrates the production of other uses of the target verb by children in the ASD and TD group. The Bayesian model (Figure 7) revealed no interaction, but main effects of Group and Verb Type. The former effect reflects the fact that children in the ASD group produced more "other" responses $(M=0.14, S D=0.34)$ than children in the TD group $(M=0.09, S D=0.29 ; p M C M C=0.027)$. The latter effect reflects the fact that, compared to the reference category of agent-patient (hit-type) verbs $(M=0.11, S D=0.32)$, children made fewer "other" responses with theme-experiencer (frighten-type) verbs $(M=0.02, S D=0.16$; $p M C M C=0.04)$ - which generally showed best performance - while experiencer-theme (see-type) verbs did not differ $(M=0.19, S D=0.40 ; p M C M C=0.21)$. The lack of an interaction suggests that these by-verb differences did not differ between the ASD and TD groups.

\section{Excluded responses}

Finally, it seemed important to verify that the pattern of results above was not unduly influenced by unequal numbers of exclusions across conditions. For example, the conclusion above that children in the ASD and TD groups produced a similar 


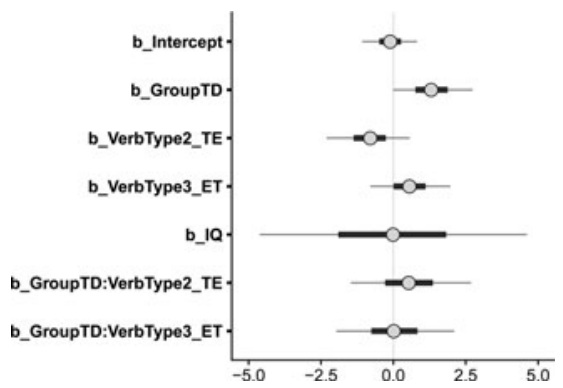

Figure 7. Bayesian model for Correct Actives

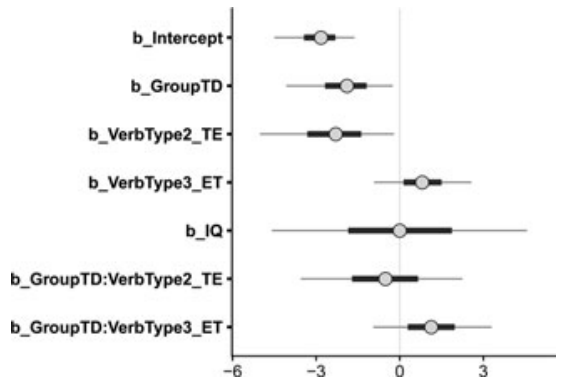

Figure 8. Bayesian model for Incorrect (other verb) uses.

proportion of correct trials would be seriously undermined if it turned out that many more trials were excluded for the former than the latter.

To investigate this possibility, we ran a final model including all responses, coded simply $(1 / 0)$ as irrelevant (i.e., excluded from the above analyses) or relevant (i.e., correct passive, reversed passive, correct active or other incorrect verb use). The effect of Group $(\mathrm{ASD} / \mathrm{TD})$ was not significant $(p M C M C=0.14)$, suggesting that the ASD $(M=0.27, S D=0.44)$ and TD groups $(M=0.23, S D=0.42)$ were broadly similar in terms of the number of excluded responses. However, compared with the reference category of agent-patient (hit-type) verbs $(M=0.10, S D=0.31)$, missing trials were slightly more common for theme-experiencer (frighten-type) verbs $(M=$ $0.32, S D=0.47 ; p M C M C=0.05)$, and experiencer-theme (see-type) verbs $(M=0.31$, $S D=0.46 ; p M C M C=0.04)$.

\section{Discussion}

The present study used a priming paradigm to compare the performance of children with ASD and an IQ-matched TD group on their production of passives. As well as comparing overall levels of correct performance and reversed passives, a further aim was to investigate whether any observed by-verb differences between agent-patient (hit-type), theme-experiencer (frighten-type) and experiencer-theme (see-type) verbs would operate similarly for the two groups, in order to further clarify the nature of any deficit observed in children with ASD.

Although the interpretation of differences by verb-type is complicated by the fact that agent-patient verbs were associated with lower levels of missing data, in general it is clear that - in line with previous studies-experiencer-theme verbs showed worst 
performance, with fewest correct passives and the most incorrect ("other") verb uses. More importantly for our purposes, in every analysis, these by-verb differences patterned in exactly the same way for ASD and TD children. No significant interaction of Verb Type by Group (ASD/TD) was observed for any dependent measure. Interestingly, this finding suggests that-despite their supposed difficulties with semantics - children with ASD (or, at least, these relatively old and high-functioning children) have a relatively sophisticated understanding of the semantics of the passive construction; summarized by Pinker, Lebeaux, and Frost (1987: 249) as:

[B] (mapped onto the surface subject [of a passive]) is in a state or circumstance characterized by [A] (mapped onto the by-object or an understood argument) having acted upon it.

This property of "affectedness" is what makes agent-patient verbs (e.g., hit) and theme-experiencer verbs (e.g., frighten) more compatible with the passive construction than are experiencer-theme verbs (e.g., see) (e.g., Ambridge, Bidgood, Pine, Rowland \& Freudenthal, 2016). The fact that, on the present findings, children with ASD are sensitive to this property suggests that, echoing findings reviewed in Naigles and Tek (2017), learning of lexical semantics is another of their relative strengths.

Crucially, however, the ASD and TD children differed with regard to what they did when they did NOT produce a correct passive (or an other/excluded response). While TD children were more likely to produce a correct active, ASD children were more likely to maintain the passive syntax of their interlocutor (as in Allen et al., 2011), but to produce an incorrect reversal (e.g., Wendy was hit by Bob, when, in fact, Wendy hit Bob). Thus, as we predicted, whilst the formal syntax of the passive seems to be relatively spared in children with $\mathrm{ASD}$, their knowledge of how this syntax maps onto thematic roles is impaired. Why, then, do children with ASD show good command of both the syntax and the lexical semantics of the passive construction, and struggle with semantics only at the level of thematic roles?

One possibility is that this deficit is not so much semantic as pragmatic. The difference between a passive sentence (e.g., Bob was hit by Wendy) and its equivalent active (e.g., Wendy hit Bob), particularly in a language such as English that lacks a natural topicalization construction, is information structure. As Pullum (2014: 64) puts it, "the denotation of the $b y$-phrase NP in a passive clause must denote something at least as new in the discourse as the subject". That is, a speaker who says Bob was hit by Wendy is talking about Bob, whereas a speaker who says Wendy hit $B o b$ is talking about Wendy. A learner who fails to appreciate this subtle information structure distinction between the active and the passive may conclude that $X$ VERBed $Y$ and $X$ was VERBed by $Y$ are just two different ways of saying the same thing, failing to appreciate that the thematic roles are reversed.

A second (and related) possibility is that this deficit is rooted not so much in semantics, but in narrative (after all, a passive sentence such as Bob was hit by Wendy is a mini narrative). Previous studies (see Naigles \& Tek, 2017, for a review), demonstrate that even high-functioning adults with ASD misinterpret story events, have difficulty naming characters, and produce story events in the wrong order (e.g., Loveland, McEvoy, Tunali \& Kelly, 1990; Colle, Baron-Cohen, Wheelwright \& van der Lely, 2008). Perhaps the failure of the present ASD group to name the characters in the required order when producing a passive construction is part and parcel of 
the same deficit. That said, it is important to stress that the study was not designed to mediate between these possibilities, and that this question must await future research.

Nevertheless, a pragmatic and/or narrative deficit could potentially explain the otherwise-surprising finding that the ASD group were more likely to produce syntactic passives (albeit often reversed ones) than the TD group. This finding is surprising given both that passives are more complex/lower frequency, and that increased production of passives presumably entails a greater priming effect (though, without active prime sentences as a control, we cannot be certain). How can a pragmatic and/or narrative deficit on the part of the ASD explain greater production of passives? Recall that the TD and ASD groups produced similar levels of correct passives, but differed in that, when they did not produce a correct passive, children in the TD group tended to produce an active, while children in the ASD group tended to produce a reversed passive. In other words, children in the TD group internalized the mini narrative (e.g., PATIENT was hit by AGENT) and recreated it using their own grammar, often defaulting to the higher-frequency active construction (Wendy hit Bob). Children in the ASD group did not internalize the mini narrative, and so were more likely to focus on maintaining the form of the experimenter's utterance, rather than its meaning.

Perhaps, then, the type of alignment observed in syntactic priming tasks is not social alignment, but a kind of formal syntactic alignment, perhaps representing implicit learning (Chang, Del, Bock \& Griffin, 2000; Rowland et al., 2012), and "social psychological factors may play a relatively minor role" (Allen et al., 2011: 546). Recall that Allen et al. (2011) found similar levels of priming for ASD and IQ-matched TD children (see also Hopkins et al., 2016, and Slocombe et al., 2013, for similar findings with children and adults with Asperger's syndrome respectively). These authors go on to suggest that the use of a game in which the child and interlocutor are adversaries (as in both Allen et al. and the present study) may be particularly unlikely to yield social alignment.

An alternative possibility is that priming does depend to a considerable extent on social alignment, but that the high-functioning ASD children included in the present study exhibited only minor social impairments. This leads to a potential criticism of the present study: perhaps at least some members of the ASD group were incorrectly classified, and did not exhibit ASD at all. While we acknowledge that it would have been preferable to administer a test such as the ADOS (which unfortunately we were not able to do), an argument against this possibility is that we observed exactly the between-groups difference with regard to reversal errors that we would expect on the basis of both previous research and theoretical accounts of language impairment in ASD. As noted earlier, a further argument against this possibility is that all children in the ASD group had undergone a lengthy and detailed diagnosis procedure (including at least one test such as the ADOS), and secured admission to a specialist school, which is routinely treated as a confirmed diagnosis, even in prevalence studies (e.g., Baron-Cohen et al., 2009).

On the other hand, it is important to acknowledge that the present study necessarily involved testing a highly unrepresentative ASD sample: high functioning children who were able to complete the priming task. Of course, this is true to some extent for all studies of linguistic abilities in ASD. Nevertheless, we need to bear in mind that such studies cannot be used to draw wide ranging claims, such as - for example - the claim that "thematic-role-learning is impaired but underlying syntax is spared in ASD". There is no justification in generalizing from a small and unrepresentative subgroup to the entire population of children with ASD. 
Future research, therefore, should attempt to extend the present findings to a more representative sample, by using experimental paradigms that place reduced task demands on children with ASD, or even play to their strengths (as Eigsti \& Bennetto, 2009, and Ambridge, Bannard \& Jackson, 2015, argue for grammaticality judgment tasks). One particularly promising paradigm is eye tracking (e.g., Brock, Norbury, Einav \& Nation, 2008; Norbury, Brock, Cragg, Einav, Griffiths \& Nation, 2009; Hahn, Snedeker \& Rabagliati, unpublished observations), in which children are not required to make any responses at all, or indeed to do anything other than pay attention to visual and linguistic stimuli. Our prediction is that, since this paradigm would allow for the inclusion of lower-functioning children with ASD, the semantic/ pragmatic/narrative deficit observed in the present study will be even greater. That is, even after (a) being primed with a passive sentence and (b) being placed in a discourse context in which the passive SUBJECT is topical, children with ASD will have greater difficulty than IQ-matched TD children in overcoming the tendency to incorrectly interpret the passive SUBJECT as an AGENT, rather than a PATIENT (see Huang, Zheng, Meng \& Snedeker, 2013, for a similar finding from an act-out study with TD children).

On the other hand, since the present findings situate the deficit with semantics/ pragmatics/narrative rather than syntactic processing, we predict that the processing difficulties associated with the passive, even for adults (e.g., Paolazzi, Grillo, Alexiadou \& Santi, 2019) will not differ for IQ matched groups of participants with and without ASD.

In conclusion, the findings of the present study suggest that the more formal elements of syntax (e.g., constituent order) are spared relative to more semantic/ pragmatic/narrative aspects, in at least high-functioning children with ASD. It is to be hoped that future studies, using both the paradigm employed here, and less demanding paradigms suitable for use with a wider range of children with ASD, will further clarify the precise nature of the linguistic impairments that typically accompany the disorder.

Acknowledgments. Ben Ambridge is Professor in the International Centre for Language and Communicative Development ( $\mathrm{LuCiD}$ ) at The University of Liverpool. The support of the Economic and Social Research Council [ES/L008955/1] is gratefully acknowledged.

Ethical approval. All procedures performed in studies involving human participants were in accordance with the ethical standards of the institutional research committee. Informed consent was obtained from all individual participants included in the study.

\section{References}

Allen, M. L., Haywood, S., Rajendran, G., \& Branigan, H. (2011). Evidence for syntactic alignment in children with autism. Developmental Science, 14(3), 540-548.

Ambridge, B., Bannard, C., \& Jackson, G. H. (2015). Is grammar spared in autism spectrum disorder? Data from judgments of verb argument structure overgeneralization errors. Journal of Autism and Developmental Disorders, 45(10), 3288-3296.

Ambridge, B., Bidgood, A., Pine, J.M., Rowland, C.F., \& Freudenthal, D. (2016). Is passive syntax semantically constrained? Evidence from adult grammaticality judgment and comprehension studies. Cognitive Science, 40(6) 1435-1459

Baird, G., Simonoff, E., Pickles, A., Chandler, S., Loucas, T., Meldrum, D., et al. (2006). Prevalence of disorders of the autism spectrum in a population cohort of children in South Thames: The Special Needs and Autism Project (SNAP). Lancet, 368(9531), 210-215. 
Baron-Cohen, S., Scott, F. J., Allison, C., Williams, J., Bolton, P., Matthews, F. E., et al. (2009). Prevalence of autism-spectrum conditions: UK school-based population study. British Journal of Psychiatry, 194(6), 500-509.

Barr, D. J., Levy, R., Scheepers, C., \& Tily, H. J. (2014). Random effects structure for confirmatory hypothesis testing: Keep it maximal. Journal of Memory and Language, 68(3), 255-278.

Brock, J., Norbury, C., Einav, S., \& Nation, K. (2008). Do individuals with autism process words in context? Evidence from language-mediated eye-movements. Cognition, 108(3), 896-904.

Bürkner, P. (2017). brms: An R Package for Bayesian Multilevel Models Using Stan. Journal of Statistical Software, 80(1), 1-28.

Chang, F., Dell, G. S., Bock, K., \& Griffin, Z. M. (2000). Structural priming as implicit learning: A comparison of models of sentence production. Journal of Psycholinguistic Research, 29(2), 217-230.

Colle, L., Baron-Cohen, S., Wheelwright, S., \& van der Lely, H.K.J. (2008) Narrative discourse in adults with high-functioning autism or Asperger syndrome. Journal of Autism and Developmental Disorders, $38,28-40$.

Eigsti, I. G., \& Bennetto, L. (2009). Grammaticality judgements in autism: Deviance or delay. Journal of Child Language, 36, 999-1021.

Eigsti, I. M., Bennetto, L., \& Dadlani, M. (2007). Beyond pragmatics: Morpho- syntactic development in autism. Journal of Autism and Developmental Disorders, 37(6), 1007-1023.

Eigsti, I. M., de Marchena, A. B., Schuh, J. M., \& Kelley, E. (2011). Language acquisition in autism spectrum disorders: A developmental review. Research in Autism Spectrum Disorders, 5(2), 681-691.

Fisher, C. I., Hahn, A. C., DeBruine, L. M., \& Jones, B. C. (2015). Women's preference for attractive makeup tracks changes in their salivary testosterone. Psychological Science, 26, 1958-1964.

Fox, D., \& Grodzinsky, Y. (1998). Children's passive: A view from the by-phrase. Linguistic Inquiry, 29, 311-332.

Gordon, P., \& Chafetz, J. (1990). Verb-based versus class-based accounts of actionality effects in children's comprehension of passives. Cognition, 36(3), 227-254.

Hahn, N., Snedeker, J., \& Rabagliati, H. (unpublished observations). Do children with autism spectrum disorders use context to resolve lexical ambiguity?

Hirsch, C., \& Wexler, K. (2006). Children's passives and their resulting interpretation. In The proceedings of the inaugural conference on generative approaches to language acquisition-North America, University of Connecticut Occasional Papers in Linguistics (Vol. 4, pp. 125-136).

Hopkins, Z., Yuill, N., \& Keller, B. (2016). Children with autism align syntax in natural conversation. Applied Psycholinguistics, 37(2), 347-370.

Horgan, D. (1978). Development of Full Passive. Journal of Child Language, 5(1), 65-80.

Huang, Y.T., Zheng, X.B., Meng, X.Z., \& Snedeker, J. (2013). Children's assignment of grammatical roles in the online processing of Mandarin passive sentences. Journal of Memory and Language, 69(4), 1-34.

Jordan, R. (1993). The nature of the linguistic and communication difficulties of children with autism. In D. J. Messer \& G. T. Turner (Eds.), Critical influences on child language acquisition and development. NY: St. Martin's Press.

Kjelgaard, M. M., \& Tager-Flusberg, H. (2001). An investigation of language impairment in autism: Implications for genetic subgroups. Language and cognitive processes, 16(2-3), 287-308.

Kogan, M. D., Blumberg, S. J., Schieve, L. A., Boyle, C. A., Perrin, J.M., Ghandour, R. M., Singh, G. K., Strickland, B. B., Trevathan, E., \& Van Dyck, P. C. (2009). Prevalence of parent-reported diagnosis of autism spectrum disorder among children in the US, 2007. Pediatrics, 124(5), 1395-1403

Loveland, K.A., McEvoy R.E., Tunali, B., \& Kelley, M.L. (1990) Narrative story telling in autism and Down's syndrome. British Journal of Developmental Psychology, 8, 9-23.

Maratsos, M. P., Fox, D. E., Becker, J. A., \& Chalkley, M. A. (1985). Semantic restrictions on children's passives. Cognition, 19(2), 167-191.

Meints, K. (1999) Protoypes and the acquisition of passives. In B. Kokinov (ed.) Perspectives on Cognitive Science, 4, 67-77. Sofia: NBU Press

Messenger, K., Branigan, H.P., McLean, J.F., \& Sorace, A. (2012). Is young children's passive syntax semantically constrained? Evidence from syntactic priming. Journal of Memory and Language, 66(4), 568-587.

Naigles, L. R. (2002). Form is easy, meaning is hard: Resolving a paradox in early child language. Cognition, 86(2), 157-199. 
Naigles, L. R., \& Tek, S. (2017). 'Form is easy, meaning is hard'revisited:(re) characterizing the strengths and weaknesses of language in children with autism spectrum disorder. Wiley Interdisciplinary Reviews: Cognitive Science, 8(4), e1438.

Naigles, L. R., Kelty, E., Jaffery, R., \& Fein, D. (2011). Abstractness and continuity in the syntactic development of young children with autism. Autism Research, 4(6), 422-437.

Norbury, C. F., Brock, J., Cragg, L., Einav, S., Griffiths, H., \& Nation, K. (2009). Eye-movement patterns are associated with communicative competence in autistic spectrum disorders. Journal of Child Psychology and Psychiatry, 50(7), 834-842.

Paolazzi, C. L., Grillo, N., Alexiadou, A., \& Santi, A. (2019). Passives are not hard to interpret but hard to remember: evidence from online and offline studies. Language, Cognition and Neuroscience, 34(8), 9911015.

Paul, R., Fisher, M., \& Cohen, D. (1988). Brief report: Sentence comprehension strategies in children with autism and specific language disorders. Journal of Autism and Developmental Disorders, 18, 669-679.

Pinker, S., Lebeaux, D. S., \& Frost, L. A. (1987). Productivity and constraints in the acquisition of the passive. Cognition, 26(3), 195-267.

Pullum, G. (2014). Fear and loathing of the English passives. Language and Communication, 37, 60-74.

Rapin, I., \& Dunn, M. (2003). Update on the language disorders of individuals on the autistic spectrum. Brain and Development, 25(3), 166-72.

Roberts, J. A., Rice, M. L., \& Tager-Flusberg, H. (2004). Tense marking in children with autism. Applied Psycholinguistics, 25(03), 429-448.

Roberts, J. E., Mirrett, P., \& Burchinal, M. (2001). Receptive and expressive communication development of young males with fragile X syndrome. Journal Information, 106(3).

Rowland, C. F., Chang, F., Ambridge, B., Pine, J. M., \& Lieven, E. V. (2012). The development of abstract syntax: Evidence from structural priming and the lexical boost. Cognition, 125(1), 49-63.

Sassenhagen, J., \& Alday, P. M. (2016). A common misapplication of statistical inference: nuisance control with null-hypothesis significance tests. Brain and Language, 162, 42-45.

Siller, M., \& Sigman, M. (2008). Modeling longitudinal change in the language abilities of children with autism: parent behaviors and child characteristics as predictors of change. Developmental psychology, 44 (6), 1691.

Slocombe, K. E., Alvarez, I., Branigan, H. P., Jellema, T., Burnett, H. G., Fischer, A., Garrod, S., \& Levita, L. (2013). Linguistic alignment in adults with and without Asperger's syndrome. Journal of autism and developmental disorders, 43(6), 1423-1436.

Sudhalter, V., \& Braine, M. D. S. (1985). How Does Comprehension of Passives Develop - a Comparison of Actional and Experiential Verbs. Journal of Child Language, 12(2), 455-470.

Swensen, L. D., Kelley, E., Fein, D., \& Naigles, L. R. (2007). Processes of language acquisition in children with autism: Evidence from preferential looking. Child development, 78(2), 542-557.

Tager-Flusberg, H. (1981). On the nature of linguistic functioning in early infantile autism. Journal of Autism and Developmental Disorders, 11(1), 45-56.

Tager-Flusberg, H. (1985). Sentence comprehension in autistic children. Applied Psycholinguistics, 2, 5-24.

Tager-Flusberg, H., Calkins, S., Nolin, T., Baumberger, T., Anderson, M., \& Chadwick-Dias, A. (1990). A longitudinal study of language acquisition in autistic and Downs syndrome children. Journal of Autism and Developmental Disorders, 20(1), 1-21.

Tager-Flusberg, H., Lord, C., \& Paul, R. (1997). Language and Communication in autism. In D. J. Cohen, \& F. R. Volkmar (Eds.), Handbook of autism and pervasive development disorders, $2^{\text {nd }}$ edition (pp. 335-365). New York: John Wiley \& Sons.

Tager-Flusberg, H., Paul, R., \& Lord, C. (2005). Language and communication in autism. Handbook of autism and pervasive developmental disorders, 1, 335-364.

Tek, S., Mesite, L., Fein, D., \& Naigles, L. (2014). Longitudinal analyses of expressive language development reveal two distinct language profiles among young children with autism spectrum disorders. Journal of autism and developmental disorders, 44(1), 75-89.

Wechsler, D. (2012). Wechsler Preschool and Primary Scale of Intelligence-Fourth Edition (WPPSI-IV): Administration and scoring manual. San Antonio, TX: The Psychological Corporation.

Zaroff, C. M., \& Uhm, S. Y. (2011). Prevalence of autism spectrum disorders and influence of country of measurement and ethnicity. Social Psychiatry and Psychiatric Epidemiology, 47(3), 395-398. 


\section{Appendix A: Diagnosis procedures}

All children in the ASD group had received a primary diagnosis of ASD, having followed one of the pathways set out below.

Children under 3;8: Social Communication Pathway. Children are referred onto this pathway by a general practitioner (GP; family doctor), speech and language therapist (SLT), community paediatrician or early years $/ 0-5$ special educational needs and disabilities (SEND) teaching team.

1. Children already in a nursery setting proceed directly to Step 2 if this is felt to be appropriate. Children not in a nursery setting attend up to six fortnightly hour-long stay and play groups run by an SLT and SLT assistants.

2. The child attends an appointment with a neurodisability paediatrician and an SLT assistant. A full medical and developmental history is taken and the child is examined.

3. An observation is conducted by an SLT, at the child's home or nursery, or at weekly hour-long playgroups run by the SLT (for up to eight weeks). The child is observed in play and play-based assessments, some based on the toddler and moule 1 of the Autism Diagnostic Observation Schedule (ADOS)

4. The child attends a 90-minute-to-two-hour appointment with the SLT who observed them and either a clinical psychologist or a paediatrician. The clinical psychologist administers a Diagnostic Interview for Social and Communication Disorders (DISCO) or an Autism Diagnostic Interview-Revised (ADI-R); or the paediatrician uses a locally-agreed proforma with similar coverage.

Children over 3;8: Social Communication Pathway. The most complex cases (and all second opinions) are undergo a three-person assessment. All three professionals are in the room with the child and family. A doctor takes the medical history, family history and some developmental history. A clinical psychologist administers a DISCO or an ADI-R. An SLT administers an ADOS. The child and family then leave the room, and the professionals discuss the information given, along with questionnaire information previously supplied by the school and family, before writing the report. In more straightforward cases, this procedure can be undertaken by a two-person team consisting of an SLT plus either a doctor or a clinical psychologist. In all cases, further assessments (e.g., school observations, cognitive assessments, further language assessments, assessments for conditions such as $\mathrm{ADHD})$ are undertaken before discussion and diagnosis, when this is felt to be necessary.

Cite this article: Ambridge B, Bidgood A, Thomas K (2021). Disentangling syntactic, semantic and pragmatic impairments in ASD: Elicited production of passives. Journal of Child Language 48, 184-201. https://doi.org/10.1017/S0305000920000215 